\title{
Revisiting two classical results on graph spectra
}

\author{
Vladimir Nikiforov \\ Department of Mathematical Sciences, University of Memphis, \\ Memphis TN 38152, USA \\ vnikifrv@memphis. edu .edu
}

Submitted: Sep 4, 2006; Accepted: Dec 18, 2006; Published: Jan 17, 2007

Mathematics Subject Classifications: 05C50

\begin{abstract}
Let $\mu(G)$ and $\mu_{\min }(G)$ be the largest and smallest eigenvalues of the adjacency matrix of a graph $G$. Our main results are:

(i) If $H$ is a proper subgraph of a connected graph $G$ of order $n$ and diameter $D$, then

$$
\mu(G)-\mu(H)>\frac{1}{\mu(G)^{2 D} n} .
$$
\end{abstract}

(ii) If $G$ is a connected nonbipartite graph of order $n$ and diameter $D$, then

$$
\mu(G)+\mu_{\min }(G)>\frac{2}{\mu(G)^{2 D} n} .
$$

For large $\mu$ and $D$ these bounds are close to the best possible ones.

Keywords: smallest eigenvalue, largest eigenvalue, diameter, connected graph, bipartite graph

\section{Introduction}

Our notation is standard (e.g., see [2], [3], and [5]). In particular, unless specified otherwise, all graphs are defined on the vertex set $[n]=\{1, \ldots, n\}$ and $\mu(G)$ and $\mu_{\min }(G)$ stand for the largest and smallest eigenvalues of the adjacency matrix of a graph $G$.

The aim of this note is to refine quantitatively two well-known results on graph spectra. The first one, following from Frobenius's theorem on nonnegative matrices, asserts that if $H$ is a proper subgraph of a connected graph $G$, then $\mu(G)>\mu(H)$. The second one, due to H. Sachs [7], asserts that if $G$ is a connected nonbipartite graph, then $\mu(G)>$ $-\mu_{\min }(G)$.

Our main result is the following theorem. 
Theorem 1 If $H$ is a proper subgraph of a connected graph $G$ of order $n$ and diameter $D$, then

$$
\mu(G)-\mu(H)>\frac{1}{\mu(G)^{2 D} n} .
$$

It can be shown that, for large $\mu$ and $D$, the right-hand of (1) gives the correct order of magnitude; examples can be constructed as in the proofs of Theorems 2 and 3 .

Theorem 2 If $G$ is a connected nonbipartite graph of order $n$ and diameter $D$, then

$$
\mu(G)+\mu_{\min }(G)>\frac{2}{\mu(G)^{2 D} n} .
$$

Moreover, for all $k \geq 3, D \geq 4$, and $n=D+2 k-1$, there exists a connected nonbipartite graph $G$ of order $n$ and diameter $D$ with $\mu(G)>k$, and

$$
\mu(G)+\mu_{\min }(G)<\frac{4}{(k-1)^{2 D-4}} .
$$

Theorem 2 shows that $\mu(G)+\mu_{\min }(G)$ can be extremely small, although $G$ is nonbipartite and connected. Here is another viewpoint to this fact.

Theorem 3 Let $0<\varepsilon<1 / 16$. For all sufficiently large $n$, there exists a connected graph $G$ of order $n$ with $\mu(G)+\mu_{\min }(G)<n^{-\varepsilon n}$ such that, to make $G$ bipartite, at least $(1 / 16-\varepsilon) n^{2}$ edges must be removed.

The picture is completely different for regular graphs. In [4] it is proved that if $G$ is a connected nonregular graph of order $n$, size $m$, diameter $D$, and maximum degree $\Delta$, then

$$
\Delta-\mu(G)>\frac{n \Delta-2 m}{n(D(n \Delta-2 m)+1)} .
$$

This result and Theorem 1 imply the following theorems; we omit their straightforward proofs.

Theorem 4 If $H$ is a proper subgraph of a connected regular graph $G$ of order $n$ and diameter $D$, then

$$
\mu(G)-\mu(H)>\frac{1}{n(D+1)} .
$$

Theorem 5 If $G$ is a connected regular nonbipartite graph of order $n$ and diameter $D$, then

$$
\mu(G)+\mu_{\min }(G)>\frac{2}{n(2 D+1)} .
$$

Theorem 6 If $G$ is a connected, nonregular, nonbipartite graph of order $n$, diameter $D$, and maximum degree $\Delta$, then

$$
\Delta+\mu_{\min }(G)>\frac{1}{n(D+1)}+\frac{1}{\mu(G)^{2 D} n} .
$$
$[1]$.

Note that the last two theorems give some fine tuning of a result of Alon and Sudakov 


\section{Proofs}

Our proof of Theorem 1 stems from a result of Schneider [8] on eigenvectors of irreducible nonnegative matrices; for graphs it reads as: if $G$ is a connected graph of order $n$ and $x_{\min }, x_{\max }$ are minimal and maximal entries of an eigenvector to $\mu(G)$, then

$$
\frac{x_{\min }}{x_{\max }} \geq \mu^{-n+1}(G)
$$

We reprove this inequality in a more flexible form that sheds some extra light on the original matrix result of Schneider as well. Hereafter we write dist $(u, v)$ for the length of a shortest path joining the vertices $u$ and $v$.

Proposition 7 If $G$ is a connected graph of order $n$ and $\left(x_{1}, \ldots, x_{n}\right)$ is an eigenvector to $\mu(G)$, then

$$
\frac{x_{i}}{x_{j}} \geq(\mu(G))^{-\operatorname{dist}(i, j)}
$$

for every two vertices $i, j \in V(G)$.

Proof Clearly we can assume that $i \neq j$. For convenience we also assume that $i=1$ and the vertices $(1, \ldots, j)$ form a path joining 1 to $j$. Then, for all $u=1, \ldots, j-1$, we have

$$
\mu x_{u}=\sum_{u v \in E(G)} x_{v} \geq x_{u+1}
$$

hence, (3) follows by multiplying all these inequalities.

Proof of Theorem 1 Since $\mu(H) \leq \mu\left(H^{\prime}\right)$ whenever $H \subset H^{\prime}$, we may assume that $H$ is a maximal proper subgraph of $G$, that is to say, $V(H)=V(G)$ and $H$ differs from $G$ in a single edge $u v$. Our proof is split into two cases: (a) $H$ connected; (b) $H$ disconnected.

Case (a): $H$ is connected.

In this case we shall prove a stronger result than required, viz.

$$
\mu(G)-\mu(H)>\frac{2}{\mu(G)^{2 D} n} .
$$

Our first goal is to prove that, for every $w \in V(H)$,

$$
\operatorname{dist}_{H}(w, u)+\operatorname{dist}_{H}(w, v) \leq 2 D \text {. }
$$

Let $w \in V(H)$ and select in $H$ shortest paths $P(u, w)$ and $P(v, w)$ joining $u$ and $v$ to $w$. Let $Q(u, x)$ and $Q(v, x)$ be the longest subpaths of $P(u, w)$ and $P(v, w)$ having no internal vertices in common. If $s \in Q(u, x)$ or $s \in Q(v, x)$, we obviously have

$$
\operatorname{dist}_{H}(w, s)=\operatorname{dist}_{H}(w, x)+\operatorname{dist}_{H}(s, x) .
$$


The paths $Q(u, x), Q(v, x)$ and the edge $u v$ form a cycle in $G$; write $k$ for its length. Assume that $\operatorname{dist}(v, x) \geq \operatorname{dist}(u, x)$ and select $y \in Q(v, x)$ with $\operatorname{dist}_{H}(x, y)=\lfloor k / 2\rfloor$. Let $R(w, y)$ be a shortest path in $G$ joining $w$ to $y$; clearly the length of $R(w, y)$ is at most $D$. If $R(w, y)$ does not contain the edge $u v$, it is a path in $H$ and, using (6), we find that

$$
\begin{aligned}
D & \geq \operatorname{dist}_{G}(w, y)=\operatorname{dist}_{H}(w, y)=\operatorname{dist}_{H}(w, x)+\lfloor k / 2\rfloor \\
& =\operatorname{dist}_{H}(w, x)+\left\lfloor\frac{\operatorname{dist}_{H}(x, u)+\operatorname{dist}_{H}(x, v)+1}{2}\right\rfloor \\
& \geq \operatorname{dist}_{H}(w, x)+\frac{\operatorname{dist}_{H}(x, u)+\operatorname{dist}_{H}(x, v)}{2}=\frac{\operatorname{dist}_{H}(w, u)+\operatorname{dist}_{H}(w, v)}{2},
\end{aligned}
$$

implying (5). Let now $R(w, y)$ contain the edge $u v$. Assume first that $v$ occurs before $u$ when traversing $R(w, y)$ from $w$ to $y$. Then

$$
\begin{aligned}
\operatorname{dist}_{H}(w, u)+\operatorname{dist}_{H}(w, v) & \leq 2 \operatorname{dist}_{H}(w, x)+\operatorname{dist}_{H}(x, u)+\operatorname{dist}_{H}(x, v) \\
& \leq 2\left(\operatorname{dist}_{H}(w, x)+\operatorname{dist}_{H}(x, v)\right)<\operatorname{dist}_{G}(w, y) \leq 2 D,
\end{aligned}
$$

implying (5). Finally, if $u$ occurs before $v$ when traversing $R(w, y)$ from $w$ to $y$, then

$$
\begin{aligned}
D & \geq \operatorname{dist}_{G}(w, y) \geq \operatorname{dist}_{H}(w, u)+1+\operatorname{dist}_{H}(v, y) \\
& =\operatorname{dist}_{H}(w, x)+\operatorname{dist}_{H}(x, u)+1+\operatorname{dist}_{H}(v, y)=\operatorname{dist}_{H}(w, x)+\lceil k / 2\rceil \\
& \geq \operatorname{dist}_{H}(w, x)+\frac{\operatorname{dist}_{H}(x, u)+\operatorname{dist}_{H}(x, v)}{2}=\frac{\operatorname{dist}_{H}(w, u)+\operatorname{dist}_{H}(w, v)}{2}
\end{aligned}
$$

implying (5). Thus, inequality (5) is proved in full.

Let now $\mathbf{x}=\left(x_{1}, \ldots, x_{n}\right)$ be a unit eigenvector to $\mu(H)$ and let $x_{w}$ be a maximal entry of $\mathbf{x}$. In view of (3) and (5), we have

$$
\frac{x_{u} x_{v}}{x_{w}^{2}} \geq \frac{1}{\mu^{\operatorname{dist}(u, w)+\operatorname{dist}(v, w)}(H)} \geq \frac{1}{\mu(H)^{2 D}} .
$$

Hence, in view of $x_{w}^{2} \geq 1 / n$, we see that

$$
\mu(G) \geq 2 \sum_{i j \in E(G)} x_{i} x_{j}=2 x_{u} x_{v}+\mu(H) \geq \frac{2 x_{w}^{2}}{\mu(H)^{2 D}}+\mu(H)>\frac{2}{\mu(H)^{2 D} n}+\mu(H),
$$

completing the proof of (4) and thus of (1).

Case (b): $H$ is disconnected.

Since $G$ is connected, $H$ is union of two connected graphs $H_{1}$ and $H_{2}$ such that $v \in H_{1}$, $u \in H_{2}$. Assume $\mu(H)=\mu\left(H_{1}\right)$, set $\left|H_{1}\right|=k, \mu=\mu\left(H_{1}\right)$, and let $\mathbf{x}=\left(x_{1}, \ldots, x_{k}\right)$ be a unit eigenvector to $\mu$. It is immediate to check that the desired inequality holds when $\left|H_{1}\right|=2,3$, so we shall assume that $k \geq 4$. Since the path of order 4 has the smallest maximal eigenvalue among all connected graphs of order at least 4, we may assume that $\mu \geq(\sqrt{5}+1) / 2$ and so $\mu^{2} \geq \mu+1$. 
Since dist $(u, w) \leq \operatorname{diam} G \leq D$ for every $w \in V\left(H_{1}\right)$, we see that dist $(v, w) \leq D-1$ for every $w \in V\left(H_{1}\right)$. On the other hand, each maximal entry of $\mathbf{x}$ is at least $k^{-1 / 2}$; hence, Proposition 7 implies that $x_{v} \geq \mu^{-D+1} k^{-1 / 2}$. Setting

$$
\mathbf{y}=\left(y_{1}, \ldots, y_{k}, y_{u}\right)=\left(x_{1}, \ldots, x_{k}, \frac{x_{v}}{\mu}\right),
$$

we see that $\|\mathbf{y}\|^{2}=1+\left(x_{v} / \mu\right)^{2}$; thus, letting $B$ be the adjacency matrix of the graph $H_{1}+u$, we have

$$
\begin{aligned}
\mu(G) & \geq \mu\left(H_{1}+u\right) \geq \frac{\langle B \mathbf{y}, \mathbf{y}\rangle}{\|\mathbf{y}\|^{2}} \geq \frac{1}{1+\left(x_{v} / \mu\right)^{2}}\left(2 y_{u} y_{v}+2 \sum_{i j \in E\left(H_{1}\right)} y_{i} y_{j}\right) \\
& =\frac{\mu^{2}}{\mu^{2}+x_{v}^{2}}\left(\frac{2 x_{v}^{2}}{\mu}+\mu\right)=\mu \frac{\mu^{2}+2 x_{v}^{2}}{\mu^{2}+x_{v}^{2}}>\mu+\mu \frac{x_{v}^{2}}{\mu^{2}+\mu}=\mu+\frac{x_{v}^{2}}{\mu+1} .
\end{aligned}
$$

To complete the proof of the theorem, observe that

$$
\frac{x_{v}^{2}}{\mu+1} \geq \frac{x_{v}^{2}}{\mu^{2}}=\frac{1}{k \mu^{2 D}}>\frac{1}{n \mu^{2 D}} .
$$

Proof of Theorem 2 Let $\mathbf{x}=\left(x_{1}, \ldots, x_{n}\right)$ be an eigenvector to $\mu_{\min }(G)$ and let $V_{1}=$ $\left\{u: x_{u}<0\right\}$. Let $H$ be the maximal bipartite subgraph of $G$, containing all edges with exactly one vertex in $V_{1}$. It is not hard to see that $H$ is connected proper subgraph of $G$, $V(H)=V(G)$, and $\mu_{\min }(H)<\mu_{\min }(G)$. Finally, let $H^{\prime}$ be a maximal proper subgraph of $G$ containing $H$. We have

$$
\mu(G)+\mu_{\min }(G) \geq \mu(G)+\mu_{\min }(H)=\mu(G)-\mu(H) \geq \mu(G)-\mu\left(H^{\prime}\right) .
$$

and (2) follows from case (a) of the proof of Theorem 1.

To construct the required example, set $G_{1}=K_{3}, G_{2}=K_{k, k}$, join $G_{1}$ to $G_{2}$ by a path $P$ of length $n-2 k-2$, and write $G$ for the resulting graph; obviously $G$ is of order $n$ and diameter $n-2 k+1$. Set $\mu=\mu(G)$ and note that $\mu(G)>k$. Let $V\left(G_{1}\right)=\left\{u_{1}, u_{2}, v_{1}\right\}$ and $P=\left(v_{1}, \ldots, v_{n-2 k-1}\right)$, where $v_{n-2 k-1} \in V\left(G_{2}\right)$. Let $\mathbf{x}$ be a unit eigenvector to $\mu(G)$ and assume that the entries $x_{1}, x_{2}, x_{3}, \ldots, x_{n-2 k+1}$ correspond to $u_{1}, u_{2}, v_{1}, \ldots, v_{n-2 k-1}$. Clearly $x_{1}=x_{2}$, and so, from $\mu x_{2}=x_{2}+x_{3}$, we find that $x_{1}=x_{2}=x_{3} /(\mu-1)$. Furthermore,

$$
\mu x_{3}=2 x_{2}+x_{4}=\frac{2 x_{3}}{\mu-1}+x_{4}<x_{3}+x_{4},
$$

and by induction we obtain $x_{i}<(\mu-1) x_{i+1}$ for all $3 \leq i \leq n-2 k$. Therefore,

$$
x_{1}=x_{2} \leq(\mu-1)^{-n+2 k+1} x_{n-2 k+1}<(k-1)^{-D+2},
$$


and by Rayleigh's principle we deduce that

$$
\mu(G)+\mu_{\min }(G) \leq 4 x_{1} x_{2}<\frac{4}{(k-1)^{2 D-4}},
$$

completing the proof.

Proof of Theorem 3 Set $r=\lceil n / 4\rceil+1, s=\lceil(1 / 2-\varepsilon) n\rceil$, select $G_{1}=K_{r, r}, G_{2}=K_{s}$, join $G_{1}$ to $G_{2}$ by a path $P$ of length $n-2 r-s+1$ and write $G$ for the resulting graph. Note first that, to make $G$ bipartite, we must remove at least

$$
\left(\begin{array}{l}
s \\
2
\end{array}\right)-\left\lfloor\frac{s^{2}}{4}\right\rfloor \geq \frac{s^{2}}{4}-\frac{s}{2}>\frac{(1 / 2-\varepsilon)^{2} n^{2}}{4}-\frac{s}{2} \geq\left(\frac{1}{16}-\varepsilon\right) n^{2}
$$

edges, for $n$ large enough. Note also that

$$
n-2\left\lceil\frac{n}{4}\right\rceil-2-\left\lceil\left(\frac{1}{2}-\varepsilon\right) n\right\rceil+1>n-\frac{n}{2}-\left(\frac{1}{2}-\varepsilon\right) n-4=\varepsilon n-4 .
$$

so the length of $P$ is greater than $\varepsilon n-4$.

Let $\mathbf{x}$ be a unit eigenvector to $\mu(G)$. Clearly the entries of $\mathbf{x}$ corresponding to vertices from $V\left(G_{1}\right) \backslash V(P)$ have the same value $\alpha$. Like in the proof of Theorem 2 , we see that

$\alpha<(n / 4)^{-\varepsilon n+5}$. Hence, by Rayleigh's principle, for $n$ large enough, we deduce that

$$
\mu(G)+\mu_{\min }(G) \leq 4 \alpha^{2}\left(\begin{array}{l}
s \\
2
\end{array}\right)<(n / 4)^{-2 \varepsilon n+10} \frac{n^{2}}{2}<(n / 4)^{-2 \varepsilon n+12}<n^{-\varepsilon n},
$$

completing the proof.

Acknowledgment: The author is indebted to Béla Bollobás for his kind support and to Sebi Cioabă for interesting discussions. Finally, the referee suggested a number of improvements, in particular, a simplification of the proof of Theorem 1.

\section{References}

[1] N. Alon, B. Sudakov, Bipartite subgraphs and the smallest eigenvalue, Combin. Probab. Comput. 9 (2000) 1-12.

[2] B. Bollobás, Modern Graph Theory, Graduate Texts in Mathematics, 184, SpringerVerlag, New York (1998), xiv+394 pp.

[3] D. Cvetković, M. Doob, H. Sachs, Spectra of Graphs, VEB Deutscher Verlag der Wissenschaften, Berlin, 1980, 368 pp.

[4] S. Cioabă, D. Gregory, V. Nikiforov, Extreme eigenvalues of nonregular graphs, to appear in J. Combin. Theory Ser B. 
[5] R. Horn, C. Johnson, Matrix Analysis, Cambridge University Press, Cambridge, 1985, xiii+561 pp.

[6] V. Nikiforov, Walks and the spectral radius of graphs, Linear Algebra Appl. 418 (2006), $257-268$

[7] H. Sachs, Beziehungen zwischen den in einem Graphen enthalteten Kreisen und seinem charakteristischen Polynom, Publ. Math. Debrecen 11 (1964) 119-134.

[8] H. Schneider, Note on the fundamental theorem on irreducible non-negative matrices, Proc. Edinburgh Math. Soc. 11 (1958/1959) 127-130. 